\title{
The Pólya-Vinogradov inequality for totally real algebraic number fields
}

by

\author{
Peter SöHne (Marburg)
}

The Pólya-Vinogradov inequality states that for any primitive character $\chi \bmod q$,

$$
\sum_{n \leq x} \chi(n) \ll q^{1 / 2} \log q .
$$

Conversely, there is a $1 \leq x \leq q$ satisfying

$$
\left|\sum_{n \leq x} \chi(n)\right| \gg q^{1 / 2}
$$

(see Montgomery and Vaughan [6]).

Here a generalization of these inequalities to totally real algebraic number fields is given. So let $K$ be a totally real field of degree $n$ over $\mathbb{Q}$ with ramification ideal $\mathfrak{d}$, absolute value of discriminant $d=N \mathfrak{d}$ and ring of integers $\mathbb{Z}_{K}$. All constants implied by the «-notation depend only on $n$, if no other dependence is explicitly noted. The nature of the difficulties in making the dependence of the constants on $n$ explicit seems to be purely technical. One has to substitute formula (6) below by a result similar to Lemma 2 of [1].

Let $\mathfrak{f} \subset \mathbb{Z}_{K}$ be an ideal, $\chi$ a primitive character of the multiplicative group $\left(\mathbb{Z}_{K} / \mathfrak{f}\right)^{*}$ extended to $\mathbb{Z}_{K}$ in the usual manner.

Finally, let $\mathbf{x} \in \mathbb{R}_{+}^{n}$ satisfy $X:=\prod_{q=1}^{n} x_{q} \geq 2$ and let $\mathbf{y} \in \mathbb{R}^{n}$.

By means of Siegel's summation formula and an additional argument Hinz [3] succeeded in showing

$$
\sum_{\substack{\nu \in \mathbb{Z}_{K} \\ 0<\nu^{(q)} \leq x_{q}}} \chi(\nu)=E(\chi) X+O_{\varepsilon}\left(N \mathfrak{f}^{1-1 /(2(n+1))} X^{\varepsilon}\right)
$$

where $\varepsilon$ is an arbitrary positive number and $E(\chi)$ equals $1 / \sqrt{d}$ if $\mathfrak{f}=\mathbb{Z}_{K}$, and 0 otherwise. 
A similar estimate was given by Lee [4] who had the exponent 1 on $N \mathfrak{f}$.

Our result is

TheOREM 1.

$$
\sum_{\substack{\nu \in \mathbb{Z}_{K} \\ y_{q}<\nu^{(q)} \leq y_{q}+x_{q}}} \chi(\nu)=E(\chi) X+O\left(d^{n / 2} N \mathfrak{f}^{1 / 2} \log ^{n}(d X)\right) .
$$

This sharpens (3) for any value of $X$ and $N \mathfrak{f}$ and is up to logarithms the same as (1). Moreover, arbitrary values of $\mathbf{y}$ may be chosen, while (3) needs $\mathbf{y}=0$

Recently Rausch ([8], (1.9)) proved this result (with constants depending on $d$ ) using a different method.

In the opposite direction we have

Theorem 2. For any $\mathbf{y} \in \mathbb{R}^{n}$ there exists $\mathbf{x} \in \mathbb{R}_{+}^{n}$, $\max _{1 \leq q \leq n} x_{q} \ll_{K}$ $N \mathfrak{f}^{1 / n}$, subject to

$$
\left|\sum_{\substack{\nu \in \mathbb{Z}_{K} \\ y_{q}<\nu^{(q)} \leq y_{q}+x_{q}}} \chi(\nu)-E(\chi) X\right| \gg_{K} N \mathfrak{f}^{1 / 2}\left(\frac{1}{\omega(2 \mathfrak{f}) \log \omega(6 \mathfrak{f})}\right) .
$$

Here $\omega(\mathfrak{a})$ denotes the number of prime divisors of $\mathfrak{a}$. In particular, the right-hand side is $\gg_{K, \varepsilon} N \mathfrak{f}^{1 / 2}(\log 2 N \mathfrak{f})^{-1-\varepsilon}$.

In the case of the ideal $\mathfrak{d} f$ being principal one has for some $\mathbf{x} \in \mathbb{R}_{+}^{n}$,

$$
\left|\sum_{\substack{\nu \in \mathbb{Z}_{K} \\ y_{q}<\nu^{(q)} \leq y_{q}+x_{q}}} \chi(\nu)-E(\chi) X\right| \geq \frac{(d N \mathfrak{f})^{1 / 2}}{(2 \pi)^{n}} .
$$

Only minor additional work has to be done to extend Theorems 1 and 2 to non-primitive characters $\chi$.

An easy corollary of the proof of Theorem 1 is given by

Proposition 1. Let $\nu_{0} \in \mathbb{Z}_{K}$. Then

$$
\begin{aligned}
\mid\left\{\nu \in \mathbb{Z}_{K} \mid \nu \equiv \nu_{0} \bmod \mathfrak{f}, y_{q}<\nu^{(q)}\right. & \left.\leq y_{q}+x_{q}, 1 \leq q \leq n\right\} \mid \\
& =\frac{X}{d^{1 / 2} N \mathfrak{f}}+O\left(d^{n / 2} \log ^{n}(X d N \mathfrak{f})\right) .
\end{aligned}
$$

The right-hand side coincides with the number of lattice points in a parallelotope (see (7) below). The problem of counting these is similar to that of counting the lattice points of a polyhedron of volume $\sim X$. For the polyhedron $\left\{\mathbf{w} \in \mathbb{R}^{n} \mid w_{j} \geq 1, \sum w_{j} \omega_{j} \leq X^{1 / n}\right\}$ it was shown by Spencer [11] that for almost all (in the sense of Lebesgue measure) coefficients $\omega_{1}, \ldots, \omega_{n}$ the remainder does not exceed $O_{\varepsilon}\left(\log ^{n+\varepsilon} X\right)$. 
In the case of $n=2$ and $\omega_{1} / \omega_{2}$ being a quadratic irrationality, Hardy and Littlewood proved that the remainder is $O(\log X)$ which is best possible ([2], Theorems A3 and A4). Thus the remainder in Proposition 1 is $O_{d, f}(\log X)$ for real-quadratic $K$. Skriganov [10] gives a proof of Proposition 1 with remainders $O_{\mathfrak{f}, d}\left(\log ^{n} X\right), n \geq 3$, and $O_{\mathfrak{f}, d}(\log X), n=2$. Nevertheless, it seems impossible to use his approach based on the inequality (3.18) of [10] to estimate character sums.

Our method of proof goes back to Pólya's original proof ([7]; see also [6]). The most important tool in it is

$$
\begin{aligned}
& \sum_{\substack{0<k \leq x \\
k \equiv l \bmod q}} 1=\left[\frac{x-l}{q}\right]-\left[\frac{-l}{q}\right] \\
&=\frac{x}{q}+\sum_{0<|m| \leq H} \frac{1}{2 \pi i m}\left(e\left(\frac{m x}{q}\right)-1\right) e\left(-\frac{m l}{q}\right) \\
& \quad+O\left(\min \left(1, \frac{1}{H\left\|\frac{x-l}{q}\right\|}+\frac{1}{H\left\|\frac{l}{q}\right\|}\right)\right),
\end{aligned}
$$

where $\|x\|:=\min (|x-k| \mid k \in \mathbb{Z})$ and $e(x):=e^{2 \pi i x}$.

Theorem 3 below gives an adequate generalization of (4).

Minkowski's convex body theorem shows that there is a $\beta^{\prime} \in \mathbb{Z}_{K}-\{0\}$ subject to

$$
\left|\beta^{\prime(q)}\right| \leq c_{1} d^{1 /(2 n)} X^{1 /(2 n)} x_{q}^{-1 / 2}, \quad 1 \leq q \leq n .
$$

$\beta:=\beta^{\prime 2}$ satisfies

$$
0<\beta^{(q)} \leq c_{1}^{2} d^{1 / n} X^{1 / n} x_{q}^{-1}, \quad 1 \leq q \leq n .
$$

By Theorem 1 of Mahler [5] there is a $\mathbb{Z}$-basis $\left\{\alpha_{1}, \ldots, \alpha_{n}\right\}$ of $\beta \mathfrak{f}$ subject to

$$
\left|\alpha_{q}^{(p)}\right| \leq c_{2} d^{1 / 2} N(\beta \mathfrak{f})^{1 / n} \leq c_{3} d N \mathfrak{f}^{1 / n}, \quad 1 \leq p, q \leq n .
$$

We use it to define the functions

$$
\alpha: \mathbb{R}^{n} \rightarrow \mathbb{R}^{n}, \quad \alpha(\mathbf{t})=\left(\sum_{q=1}^{n} t_{q} \alpha_{q}^{(p)}\right)_{p=1}^{n} \quad\left(\text { thus } \alpha\left(\mathbb{Z}^{n}\right)=\beta \mathfrak{f}\right)
$$

and

$$
\eta:=\alpha^{-1 \top}: \mathbb{R}^{n} \rightarrow \mathbb{R}^{n} \quad\left(\text { thus } \eta\left(\mathbb{Z}^{n}\right)=1 /(\mathfrak{d} \beta \mathfrak{f})\right) .
$$

Moreover, for $\mathbf{u}, \mathbf{v}, \mathbf{z} \in \mathbb{R}^{n}$ with

$$
0<v_{p} \leq 2 c_{1}^{2} d^{1 / n} X^{1 / n}, \quad 1 \leq p \leq n,
$$

we define

$$
F(\mathbf{u}, \mathbf{z}):=F(\mathbf{u}, \mathbf{z} ; \mathbf{v}, \alpha):=\left|\left\{\mathbf{m} \in \mathbb{Z}^{n} \mid z_{p}<\alpha^{(p)}(\mathbf{m}+\mathbf{u}) \leq z_{p}+v_{p}\right\}\right| .
$$


In Sections 1-3 we fix $\mathbf{z}$ and work with the Fourier series of $F$ with respect to $\mathbf{u}$. This will prove Theorem 1 .

In Section $4, \mathbf{u}$ is fixed and the Fourier expansion of $F$ with respect to $\mathbf{z}$ is used to derive lower bounds. Here only $L^{2}$-convergence of the series is needed, so that the proof is easily compared to that of the upper bounds requiring a result similar to (4).

We make use of the notations

$$
|\mathbf{t}|_{\infty}:=\max \left(\left|t_{j}\right| \mid 1 \leq j \leq k\right) \quad \text { and } \quad\langle\mathbf{s}, \mathbf{t}\rangle:=\sum_{j=1}^{k} s_{j} t_{j}, \quad \mathbf{s}, \mathbf{t} \in \mathbb{R}^{k} ;
$$

in particular,

$$
|\nu|_{\infty}=\max \left(\left|\nu^{(q)}\right| \mid 1 \leq q \leq n\right) \quad \text { and } \quad\langle\nu, \mu\rangle=S(\nu \mu) \quad \text { for } \nu, \mu \in K .
$$

\section{Preliminary lemmas. First we need}

LEMma 1. For a natural number $N$ and reals $v<w$ one has

$$
\int_{v}^{w} \sum_{N<|k| \leq 2 N} e(k t) d t \ll \frac{1}{N} \min \left(\frac{1}{\|v\|}+\frac{1}{\|w\|}, N\right) .
$$

Proof. Obviously, it suffices to prove the lemma assuming $v, w \notin \mathbb{Z}$. The integral equals

$$
\sum_{N<|k| \leq 2 N} \frac{1}{2 \pi i k}(e(k w)-e(k v))
$$

and is, therefore, by trivial estimation, $\ll 1$, and is

$$
\ll \frac{1}{N} \min \left(\frac{1}{\|v\|}+\frac{1}{\|w\|}\right)
$$

by use of partial summation and of $\sum_{a<k<b} e(k t) \ll 1 /\|t\|$.

Lemma 2. Let $M, T \geq 2, C \geq 1$ and $\beta \in \mathbb{R}$. Then

$$
\begin{aligned}
\int_{-C}^{C} \min \left(\frac{1}{\|t\|}, M\right) \min \left(\frac{1}{|t+\beta|}, T\right) d t & \\
& \ll \log (M T) \sum_{|m| \leq 2 C} \min \left(\frac{1}{|m+\beta|}, M T\right) .
\end{aligned}
$$

Proof. The left-hand side is less than

$$
\sum_{|m| \leq 2 C} \int_{m-1 / 2}^{m+1 / 2} \min \left(\frac{1}{|t-m|}, M\right) \min \left(\frac{1}{|t+\beta|}, T\right) d t .
$$

For fixed $m$, the integral can be estimated in a trivial way by $M T$. 
For $|m+\beta| \geq 1$ one has

$$
\min \left(\frac{1}{|t+\beta|}, T\right) \ll \min \left(\frac{1}{|m+\beta|}, T\right) \quad(m-1 / 2 \leq t \leq m+1 / 2)
$$

and

$$
\int_{m-1 / 2}^{m+1 / 2} \min \left(\frac{1}{|t-m|}, M\right) d t=2 \log \left(\frac{e M}{2}\right) \ll \log (M T) .
$$

Otherwise, let

$$
\left.I_{1}:=\right] m-\frac{1}{M T}, m+\frac{1}{M T}[\cup]-\beta-\frac{1}{M T},-\beta+\frac{1}{M T}[
$$

and

$$
I_{2}:=[m-1 / 2, m+1 / 2]-I_{1} .
$$

The integral taken over $I_{1}$ does not exceed $4 \ll \min (1 /|m+\beta|, T) . I_{2}$ is the union of at most 3 subintervals. Let $\left[v_{1}, v_{2}\right]$ be one of them. Then

$$
\int_{v_{1}}^{v_{2}} \frac{d t}{|t-m||t+\beta|}=\left|\int_{v_{1}}^{v_{2}} \frac{d t}{(t-m)(t+\beta)}\right|
$$

(note that the integrand does not change its sign on $\left[v_{1}, v_{2}\right]$ )

$$
\begin{aligned}
& =\left|\frac{1}{m+\beta} \int_{v_{1}}^{v_{2}}\left(\frac{1}{t-m}-\frac{1}{t+\beta}\right) d t\right| \\
& \ll \frac{1}{|m+\beta|} \log (M T) .
\end{aligned}
$$

Lemma 3. Let $k \in \mathbb{N}, \mathbf{a} \in(\mathbb{R}-\{0\})^{k}, M \geq 2, \beta \in \mathbb{R}$ and $C \geq 1$. Assume

$$
T \geq M+2 \max _{1 \leq j \leq k}\left(\left|a_{j}\right|+\left|a_{j}^{-1}\right|\right) .
$$

Then

$$
\begin{aligned}
\int_{[-C, C]^{k}} \prod_{q=1}^{k} \min ( & \left.\frac{1}{\left\|t_{q}\right\|}, M\right) \min \left(\frac{1}{|\langle\mathbf{a}, \mathbf{t}\rangle+\beta|}, T\right) d t \\
& \ll_{k}(\log T)^{k} \sum_{\substack{\mathbf{m} \in \mathbb{Z}^{k} \\
|\mathbf{m}|_{\infty} \leq 2^{k} C}} \min \left(\frac{1}{|\langle\mathbf{a}, \mathbf{m}\rangle+\beta|}, T M^{k}\right) .
\end{aligned}
$$


Proof. We use induction on $k$ and the formula

$$
\begin{aligned}
\int_{-C}^{C} \min \left(\frac{1}{\left\|t_{k}\right\|}, M\right) \min \left(\frac{1}{|\langle\mathbf{a}, \mathbf{t}\rangle+\beta|}, T\right) d t_{k} \\
=\frac{1}{\left|a_{k}\right|} \int_{-C}^{C} \min \left(\frac{1}{\left\|t_{k}\right\|}, M\right) \\
\quad \times \min \left(\frac{1}{\left|t_{k}+\left(\sum_{j \leq k-1} t_{j} a_{j}+\beta\right) a_{k}^{-1}\right|}, T\left|a_{k}\right|\right) d t_{k} \\
\ll \log T \sum_{\left|m_{k}\right| \leq 2 C} \min \left(\frac{1}{\left|\sum_{j \leq k-1} t_{j} a_{j}+\left(a_{k} m_{k}+\beta\right)\right|}, T M\right) \\
\forall\left(t_{1}, \ldots, t_{k-1}\right) \in[-C, C]^{k-1}
\end{aligned}
$$

by Lemma 2 .

2. Fourier expansion of $F(\cdot, \mathbf{z})$. Obviously, one has

$$
F(\cdot, \mathbf{z})=\sum_{\mathbf{n} \in \mathbb{Z}^{n}} a_{\mathbf{n}} e(\langle\mathbf{n}, \cdot\rangle) \quad \text { in } L^{2}\left([0,1]^{n}\right),
$$

where

$$
\begin{aligned}
a_{\mathbf{n}}= & a_{\mathbf{n}}(\mathbf{z} ; \mathbf{v}, \alpha)=\int_{[0,1]^{n}} F(\mathbf{u}, \mathbf{z}) e(-\langle\mathbf{n}, \mathbf{u}\rangle) d \mathbf{u} \\
= & \sum_{\substack{\mathbf{m} \in \mathbb{Z}^{n} \\
\mathbf{m}+\mathbf{u} \in V}} \int_{\substack{[0,1]^{n}\\
}} e(-\langle\mathbf{n}, \mathbf{m}+\mathbf{u}\rangle) d \mathbf{u}=\int_{V} e(-\langle\mathbf{n}, \mathbf{u}\rangle) d \mathbf{u}, \\
& V:=\alpha^{-1}\left(\underset{p=1}{\left.\left.\underset{X}{X}] z_{p}, z_{p}+v_{p}\right]\right)} .\right.
\end{aligned}
$$

For brevity, let

$$
\text { (10) }\left\{\begin{array}{l}
\tau:=c_{4} d^{n / 2}\left(\left(\frac{X}{N \mathfrak{f}}\right)^{1 / n}+1\right), \\
\tau^{\prime}:=c_{5} d^{n}\left(X^{1 / n}+N \mathfrak{f}^{1 / n}\right), \\
\mathbf{k} \in \mathbb{Z}^{n} \text { given by } k_{q}:=\left[\left(\alpha^{-1}(\mathbf{z})\right)^{(q)}\right], \text { thus }\left|\mathbf{k}-\alpha^{-1}(\mathbf{z})\right|_{\infty} \leq 1 .
\end{array}\right.
$$

Lemma $4 . \mathbf{t} \in V, \mathbf{u} \in[0,1]^{n} \Rightarrow|\mathbf{t}-\mathbf{u}-\mathbf{k}|_{\infty} \leq \tau$. 
Proof. Cramer's rule and (6) imply

$$
\begin{aligned}
\max _{|\mathbf{w}|_{\infty} \leq 1}\left|\alpha^{-1}(\mathbf{w})\right|_{\infty} & \ll\left|\operatorname{det}\left(\alpha_{p}^{(q)}\right)\right|^{-1}\left(\max _{p, q}\left|\alpha_{p}^{(q)}\right|\right)^{n-1} \\
& \ll d^{n / 2-1} N(\beta \mathfrak{f})^{-1 / n} \ll d^{n-1} N \mathfrak{f}^{-1 / n} .
\end{aligned}
$$

This proves the assertion since (7) and (6) yield

$$
\begin{aligned}
|\alpha(\mathbf{t}-\mathbf{u}-\mathbf{k})|_{\infty} & =\left|(\alpha(\mathbf{t})-\mathbf{z})+\alpha\left(\alpha^{-1}(\mathbf{z})-\mathbf{u}-\mathbf{k}\right)\right|_{\infty} \\
& \leq|\mathbf{v}|_{\infty}+\max _{|\mathbf{w}|_{\infty} \leq 2}|\alpha(\mathbf{w})|_{\infty} \\
& \ll(d X)^{1 / n}+d N \mathfrak{f}^{1 / n} .
\end{aligned}
$$

Proposition 2. Let $N \geq 2 \tau^{\prime}$ and $\mathbf{u} \in[0,1]^{n}, \varrho:=\alpha(\mathbf{u})$. Then

$$
\begin{aligned}
& \sum_{\substack{\mathbf{n} \in \mathbb{Z}^{n} \\
N<|\mathbf{n}|_{\infty} \leq 2 N}} a_{\mathbf{n}} e(\langle\mathbf{n}, \mathbf{u}\rangle) \mid \\
& \ll \frac{\log ^{n} N}{N} d N \mathfrak{f}^{1 / n} \sum_{\substack{\nu \in \beta \mathfrak{f} \\
|\nu-\mathbf{z}|_{\infty} \leq \tau^{\prime}}} \sum_{c=0}^{1} \sum_{p=1}^{n} \min \left(\frac{1}{\left|z_{p}+c v_{p}-\varrho^{(p)}-\nu^{(p)}\right|}, N^{n} \tau\right) .
\end{aligned}
$$

Proof. Our approach should be compared to the proof of Theorem 1 of Tatuzawa [12]. We divide the left-hand side into $2^{n}-1$ subsums taken over the sets

$$
W_{I}=\left\{\mathbf{n} \in \mathbb{Z}^{n}|N<| n_{q}|\leq 2 N \forall q \in I,| n_{q} \mid \leq N \forall q \notin I\right\}
$$

corresponding to the nonempty sets $I \subset\{1, \ldots, n\}$. Let $I$ be one of these sets; to simplify the notation we assume $n \in I$.

(9) leads to

$$
\begin{aligned}
& \left|\sum_{\mathbf{n} \in W_{I}} a_{\mathbf{n}} e(\langle\mathbf{n}, \mathbf{u}\rangle)\right|=\left|\int_{V} \sum_{\mathbf{n} \in W_{I}} e(\langle\mathbf{n}, \mathbf{u}-\mathbf{t}\rangle) d \mathbf{t}\right| \\
& =\left|\int_{V} \prod_{p \in I} \sum_{N<\left|n_{p}\right| \leq 2 N} e\left(n_{p}\left(u_{p}-t_{p}\right)\right) \prod_{p \notin I I} \sum_{\left|n_{p}\right| \leq 2 N} e\left(n_{p}\left(u_{p}-t_{p}\right)\right) d \mathbf{t}\right| \\
& \ll \int_{[-\tau, \tau]^{n-1}} \prod_{p=1}^{n-1} \min \left(\frac{1}{\left\|s_{p}\right\|}, N\right) \\
& \quad \times\left.\right|_{\left\{s_{n} \mid\left(s_{1}, \ldots, s_{n}\right)^{\top}+\mathbf{u}+\mathbf{k} \in V\right\}} \sum_{N<\left|n_{n}\right| \leq 2 N} e\left(n_{n} s_{n}\right) d s_{n} \mid d^{n-1} \mathbf{s}
\end{aligned}
$$

by means of the substitution $\mathbf{s}=\mathbf{t}-\mathbf{u}-\mathbf{k}$ and of Lemma 4 . 
Now $\overline{\left\{s_{n} \mid\left(s_{1}, \ldots, s_{n}\right)+\mathbf{u}+\mathbf{k} \in V\right\}}=:\left[\xi_{1}, \xi_{2}\right]$ is an interval, and

$$
\begin{aligned}
& \alpha^{(p)}\left(\left(s_{1}, \ldots, s_{n-1}, \xi_{j}\right)+\mathbf{u}+\mathbf{k}\right)=z_{p}+c v_{p} \\
& \quad \Rightarrow \xi_{j}=\left(z_{p}+c v_{p}-\varrho^{(p)}-\alpha^{(p)}(k)-\sum_{j=1}^{n-1} s_{j} \alpha_{j}^{(p)}\right) \alpha_{n}^{(p)}{ }^{-1}=: \xi_{c p}(\mathbf{s})
\end{aligned}
$$

for some $c \in\{0,1\}, p \in\{1, \ldots, n\}$.

Since

$$
\begin{aligned}
\xi_{c p}(\mathbf{s}) & =\left(c v_{p}+\alpha^{(p)}\left(\alpha^{-1}(\mathbf{z})-\mathbf{u}-\mathbf{k}+O(\tau)\right)\right) \alpha_{n}^{(p)^{-1}} \\
& \ll\left((d X)^{1 / n}+d N \mathfrak{f}^{1 / n} \tau\right)\left|\prod_{q \neq p} \alpha_{n}^{(q)} N \alpha_{n}^{-1}\right| \\
& \ll\left(X^{1 / n}+N \mathfrak{f}^{1 / n} \tau\right) d^{n / 2} N \mathfrak{f}^{-1 / n} \\
& \ll d^{n / 2} \tau \quad \text { by }(6) \&(7),
\end{aligned}
$$

the inner integral is, by Lemma 1 ,

$$
\begin{aligned}
& \ll \frac{1}{N} \sum_{c, p} \min \left(\frac{1}{\left\|\xi_{c p}(\mathbf{s})\right\|}, N\right) \\
& \ll \frac{1}{N} \sum_{c, p} \sum_{\left|m_{n}\right| \leq c_{6} d^{n / 2} \tau} \min \left(\frac{1}{\left|\xi_{c p}(\mathbf{s})-m_{n}\right|}, N\right) .
\end{aligned}
$$

This gives

$$
\begin{aligned}
& \left|\sum_{\mathbf{n} \in W_{I}} a_{\mathbf{n}} e(\langle\mathbf{n}, \mathbf{u}\rangle)\right| \\
& \ll \frac{1}{N} \sum_{c, p} \sum_{\left|m_{n}\right| \leq c_{6} d^{n / 2} \tau} \int_{[-\tau, \tau]^{n-1}} \prod_{j=1}^{n-1} \min \left(\frac{1}{\left|s_{j}\right|}, N\right) \\
& \quad \times \min \left(\frac{1}{\left|\xi_{c p}(\mathbf{s})-m_{n}\right|}, N\right) d^{n-1} \mathbf{s} \\
& \ll \frac{\log ^{n} N}{N} \sum_{c, p} \sum_{|\mathbf{m}|_{\infty} \leq c_{7} d^{n / 2} \tau} \min \left(\frac{1}{\left|\xi_{c p}\left(\left(m_{1}, \ldots, m_{n-1}\right)^{\top}\right)-m_{n}\right|}, \tau N^{n}\right)
\end{aligned}
$$

by Lemma 3, which is applicable because (6) imply

$$
\begin{aligned}
\max _{h, i, j}\left|\left(\alpha_{j} / \alpha_{h}\right)^{(i)}\right| & =\max _{h, i, j}\left|\alpha_{j}^{(i)} \prod_{k \neq i} \alpha_{h}^{(k)} / N \alpha_{h}\right| \\
& \ll\left(d^{1 / 2} N \mathfrak{f}^{1 / n}\right)^{n} N \beta \mathfrak{f}^{-1} \ll d^{n / 2} \ll \tau .
\end{aligned}
$$


Now

$$
\begin{aligned}
\left(\xi_{c p}\left(\left(m_{1}, \ldots, m_{n-1}\right)^{\top}\right)-m_{n}\right) \alpha_{n}^{(p)} & =z_{p}+c v_{p}-\varrho^{(p)}-\alpha^{(p)}(\mathbf{k})-\alpha^{(p)}(\mathbf{m}) \\
& =z_{p}+c v_{p}-\varrho^{(p)}-\nu^{(p)},
\end{aligned}
$$

where $\nu:=\alpha(\mathbf{k}+\mathbf{m}) \in \beta \mathfrak{f}$ satisfies

$$
|\nu-\mathbf{z}|_{\infty}=\left|\alpha\left(\mathbf{k}-\alpha^{-1}(\mathbf{z})+\mathbf{m}\right)\right|_{\infty} \leq \max \left(\left.|\alpha(\mathbf{t})|_{\infty}|| \mathbf{t}\right|_{\infty} \leq c_{7} \tau d^{n / 2}\right) \leq \tau^{\prime}
$$

for sufficiently large $c_{5}$.

Moreover, $\mathbf{m} \rightarrow \nu$ is injective, and the assertion follows since $\left|\alpha_{n}^{(p)}\right| \ll$ $d N \mathfrak{f}^{1 / n}$.

Proposition 3. Let $N \geq 2 \tau$. Then

$$
\begin{aligned}
F(\mathbf{u}, \mathbf{z})= & \sum_{\substack{\mathbf{n} \in \mathbb{Z}^{n} \\
|\mathbf{n}|_{\infty} \leq N}} a_{\mathbf{n}} e(\langle\mathbf{n}, \mathbf{u}\rangle) \\
& +O\left(\frac{\log ^{n} N}{N} d N \mathfrak{f}^{1 / n} \sum_{\substack{\nu \in \beta \mathfrak{f} \\
|\nu-\mathbf{z}|_{\infty} \leq \tau^{\prime}}} \sum_{c=0}^{1} \sum_{p=1}^{n} \frac{1}{\left|z_{p}+c v_{p}+\alpha^{(p)}(\mathbf{u})-\nu^{(p)}\right|}\right)
\end{aligned}
$$

for any $\mathbf{u} \in[0,1]^{n}$.

R e m a r k. For certain values of $\mathbf{u}$ the expression inside $O(\cdot)$ is not finite. It is easy to show (but not needed in this paper) that the remainder does not exceed

$$
O\left(\left(\frac{X}{N \mathfrak{f}}\right)^{1-1 / n}+d^{n / 2} \log ^{n} N X\right) .
$$

One has to combine Lemma 5 below and a result similar to Hilfssatz 10 of [9].

Proof of Proposition 3. Define

$$
\begin{aligned}
K:=\left\{\mathbf{u} \in[0,1]^{n}|\exists \nu \in \beta \mathfrak{f},| \nu-\left.\mathbf{z}\right|_{\infty} \leq \tau^{\prime},\right. \\
\left.\exists c, p: z_{p}+c v_{p}=\nu^{(p)}+\alpha^{(p)}(\mathbf{u})\right\}, \\
G:=[0,1]^{n}-K \quad \text { and } \quad F_{m}(\mathbf{u}):=\sum_{\substack{\mathbf{n} \in \mathbb{Z}^{n} \\
N<|\mathbf{n}|_{\infty} \leq 2^{m} N}} a_{\mathbf{n}} e(\langle\mathbf{n}, \mathbf{u}\rangle) .
\end{aligned}
$$

Proposition 1 yields, for $\mathbf{u} \in G$,

$$
\begin{aligned}
& \sum_{m=1}^{\infty}\left|F_{m}(\mathbf{u})\right| \\
& \ll \sum_{m=1}^{\infty} \frac{\log ^{n}\left(2^{m} N\right)}{2^{m} N} d N \mathfrak{f}^{1 / n} \sum_{c, p} \sum_{|\nu-\mathbf{z}|_{\infty} \leq \tau^{\prime}} \frac{1}{\left|z_{p}+c v_{p}-\alpha^{(p)}(\mathbf{u})-\nu^{(p)}\right|}
\end{aligned}
$$




$$
\ll \frac{\log ^{n} N}{N} d N \mathfrak{f}^{1 / n} \sum_{\substack{\nu \in \beta \mathfrak{f} \\|\nu-\mathbf{z}|_{\infty} \leq \tau^{\prime}}} \sum_{c=0}^{1} \sum_{p=1}^{n} \frac{1}{\left|z_{p}+c v_{p}+\alpha^{(p)}(\mathbf{u})-\nu^{(p)}\right|} .
$$

Therefore, $\sum_{m=1}^{\infty} F_{m}$ converges uniformly on any compact set $\widetilde{G} \subset G$. It coincides by (8) with

$$
F(\cdot, \mathbf{z})-\sum_{\substack{\mathbf{n} \in \mathbb{Z}^{n} \\|\mathbf{n}|_{\infty} \leq N}} a_{\mathbf{n}} e(\langle\mathbf{n}, \cdot\rangle) \quad \text { in } L^{2}(\widetilde{G}) .
$$

Since $K$ is closed and both the functions are continuous, equality holds at every point of $G$, and the assertion follows (of course it is trivial for $\mathbf{u} \in K$ ).

3. Upper bounds. From Proposition 3 we derive our generalization of (4):

TheOREM 3. There are complex numbers $b_{\mathbf{n}}=b_{\mathbf{n}}(\mathbf{x}, \mathbf{y}, \alpha)$ satisfying

$$
\left|b_{\mathbf{n}}\right| \ll \frac{1}{\sqrt{d} N \beta \mathfrak{f}} \prod_{p=1}^{n} \min \left(\frac{1}{\left|\eta^{(p)}(\mathbf{n})\right|}, X^{1 / n}\right)
$$

and

$$
\begin{aligned}
\left|\left\{\nu \in \mathbb{Z}_{K} \mid \nu \equiv \nu_{0} \bmod \mathfrak{f}, y_{q}<\nu^{(q)} \leq y_{q}+x_{q}, 1 \leq q \leq n\right\}\right| \\
=\frac{X}{\sqrt{d} N \mathfrak{f}}+\sum_{\substack{\mathbf{n} \in \mathbb{Z}^{n} \\
0<|\mathbf{n}|_{\infty} \leq N}} b_{\mathbf{n}} e\left(S\left(\eta(\mathbf{n}) \beta \nu_{0}\right)\right)+O\left(N^{-1 / 3}\right)
\end{aligned}
$$

for any $\nu_{0} \in \mathbb{Z}_{K}$ and any $N \geq c_{8}(d N \mathfrak{f} X)^{c_{9}}$.

Pr o of. Let $\widetilde{z}_{q}:=\beta^{(q)} y_{q}, \widetilde{v}_{q}:=\beta^{(q)} x_{q}, 1 \leq q \leq n$. For different integers $\nu_{1}, \nu_{2}$ of $K$ satisfying $\left|\nu_{j}-\widetilde{\mathbf{z}}\right|_{\infty} \leq 2 \tau^{\prime}$,

$$
\min _{1 \leq p \leq n}\left|\nu_{1}^{(p)}-\nu_{2}^{(p)}\right| \geq\left|N\left(\nu_{1}-\nu_{2}\right)\right| /\left|\nu_{1}-\nu_{2}\right|_{\infty}^{n-1} \geq\left(4 \tau^{\prime}\right)^{1-n}
$$

Thus at least one of the intervals

$$
] \widetilde{z}_{p}+c \widetilde{v}_{p}-\left(8 \tau^{\prime}\right)^{1-n}, \widetilde{z}_{p}+c \widetilde{v}_{p}\right] \text { and }\right] \widetilde{z}_{p}+c \widetilde{v}_{p}, \widetilde{z}_{p}+c \widetilde{v}_{p}+\left(8 \tau^{\prime}\right)^{1-n}\right]
$$

does not contain the $p$ th conjugate of any $\nu \in \mathbb{Z}_{K},|\nu-\mathbf{z}|_{\infty} \leq \tau^{\prime}$.

This allows us to choose $a_{c p}, b_{c p} \in\{0,1\}$ so that

and

$$
z_{p}:=\widetilde{z}_{p}+(-1)^{a_{c p}}\left(8 \tau^{\prime}\right)^{1-n} N^{-1 / 3}
$$

satisfy

$$
v_{p}:=\widetilde{v}_{p}+\left(\widetilde{z}_{p}-z_{p}\right)+(-1)^{b_{c p}}\left(8 \tau^{\prime}\right)^{1-n} N^{-1 / 3}
$$

$\left|z_{p}+c v_{p}-\nu^{(p)}\right| \geq\left(8 \tau^{\prime}\right)^{1-n} N^{-1 / 3} \quad \forall \nu \in \mathbb{Z}_{K}:|\nu-\mathbf{z}|_{\infty} \leq \tau^{\prime} \forall c \in\{0,1\} \forall p$ 
and (since all elements of the counted sets are integers $\nu$ subject to $|\nu-\mathbf{z}|_{\infty}$ $\left.\leq 2 \tau^{\prime}\right)$

$$
\begin{aligned}
F\left(\alpha^{-1}\left(\beta \nu_{0}\right), \mathbf{z} ; \mathbf{v}, \alpha\right) & =F\left(\alpha^{-1}\left(\beta \nu_{0}\right), \widetilde{\mathbf{z}} ; \widetilde{\mathbf{v}}, \alpha\right) \\
& =\left|\left\{\mathbf{m} \in \mathbb{Z}^{n} \mid \widetilde{z}_{p}<\alpha^{(p)}\left(\mathbf{m}+\alpha^{-1}\left(\beta \nu_{0}\right)\right) \leq \widetilde{z}_{p}+\widetilde{v}_{p}\right\}\right| \\
& =\left|\left\{\mu \in \beta \mathfrak{f} \mid \beta^{(p)} y_{p}<\left(\mu+\beta \nu_{0}\right)^{(p)} \leq \beta^{(p)}\left(y_{p}+x_{p}\right)\right\}\right| \\
& =\left|\left\{\nu \in \mathbb{Z}_{K} \mid \nu \equiv \nu_{0} \bmod \mathfrak{f}, y_{p}<\nu^{(p)} \leq y_{p}+x_{p}\right\}\right| .
\end{aligned}
$$

(7) holds because of (5) and of $v_{p}=\widetilde{v}_{p}+O\left(N^{-1 / 3}\right)=\beta^{(p)} x_{p}+O\left(N^{-1 / 3}\right)$.

Thus Proposition 3 can be used to obtain

$$
\begin{aligned}
\mid\{\nu \in & \left.\mathbb{Z}_{K} \mid \nu \equiv \nu_{0} \bmod \mathfrak{f}, y_{p}<\nu^{(p)} \leq y_{p}+x_{p}\right\} \mid \\
= & \sum_{\substack{\mathbf{n} \in \mathbb{Z}^{n} \\
|\mathbf{n}|_{\infty} \leq N}} a_{\mathbf{n}}(\mathbf{z}, \mathbf{v}, \alpha) e\left(\left\langle\mathbf{n}, \alpha^{-1}\left(\beta \nu_{0}\right)\right\rangle\right) \\
& +O\left(\frac{\log ^{n} N}{N} d N \mathfrak{f}^{1 / n} \sum_{\substack{\nu \in \beta \mathfrak{f} \\
|\nu-\mathbf{z}|_{\infty} \leq \tau^{\prime}}}\left(\tau^{\prime 1-n} N^{-1 / 3}\right)^{-1}\right) \\
& =\sum_{\substack{\mathbf{n} \in \mathbb{Z}^{n} \\
|\mathbf{n}|_{\infty} \leq N}} a_{\mathbf{n}}(\mathbf{z}, \mathbf{v}, \alpha) e\left(\left\langle\eta(\mathbf{n}), \beta \nu_{0}\right\rangle\right)+O\left(\frac{\log ^{n} N}{N} d^{1 / 2} \tau^{\prime 2 n-1} N^{1 / 3}\right)
\end{aligned}
$$

by use of

$$
\begin{aligned}
\left|\left\{\nu \in \beta \mathfrak{f}|| \nu-\left.\mathbf{z}\right|_{\infty} \leq \tau^{\prime}\right\}\right| & =\left|\left\{\mathbf{m} \in \mathbb{Z}^{n}|| \alpha(\mathbf{m})-\left.\alpha(\mathbf{z})\right|_{\infty} \leq \tau^{\prime}\right\}\right| \\
& \leq \operatorname{Vol}\left(\left.\mathbf{t} \in \mathbb{R}^{n}|| \alpha(\mathbf{t})\right|_{\infty} \leq 2 \tau^{\prime}\right) \ll \frac{\tau^{\prime n}}{\sqrt{d} N \beta \mathfrak{f}} .
\end{aligned}
$$

For sufficiently large $c_{9}$ the remainder is $\ll N^{-1 / 3}$ (see (10)).

Moreover, by means of the substitution $\mathbf{t}=\alpha(\mathbf{v}),(9)$ gives

$$
b_{\mathbf{n}}:=a_{\mathbf{n}}(\mathbf{z}, \mathbf{v}, \alpha)=\frac{1}{\sqrt{d} N \beta \mathfrak{f}} \prod_{p=1}^{n} \int_{z_{p}}^{z_{p}+v_{p}} e\left(-\eta^{(p)}(\mathbf{n}) t_{p}\right) d t_{p},
$$

which shows the estimate for the $b_{\mathbf{n}}, \mathbf{n} \neq \mathbf{0}$, and

$$
\begin{aligned}
b_{\mathbf{0}}: & =\frac{1}{\sqrt{d} N \beta \mathfrak{f}} \prod_{p=1}^{n} v_{p}=\frac{1}{\sqrt{d} N \beta \mathfrak{f}} \prod_{p=1}^{n}\left(\widetilde{v}_{p}+O\left(\tau^{1-n} N^{-1 / 3}\right)\right) \\
& =\frac{1}{\sqrt{d} N \beta \mathfrak{f}}\left(\prod_{p=1}^{n} \beta^{(p)} x_{p}+O\left(\left(\frac{(d X)^{1 / n}}{\tau^{\prime}}\right)^{n-1} N^{-1 / 3}\right)\right) \quad \text { by }(5) \\
& =\frac{N \beta X}{\sqrt{d} N \beta \mathfrak{f}}+O\left(N^{-1 / 3}\right) \quad \text { by }(10) .
\end{aligned}
$$


Lemma 5. Let $\mathfrak{c}$ denote a (not necessarily integral) ideal of $K$ and let $M \geq 2+N \mathfrak{c}$. Then

$$
\sum_{\substack{\gamma \in \mathfrak{c} \\ 0<|\gamma|_{\infty} \leq M}} \frac{1}{|N \gamma|} \ll d^{(n-1) / 2} N \mathfrak{c}^{-1}(\log M)^{n} .
$$

Pr o of. Given $\mathbf{z} \in \mathbb{R}_{+}^{n}, Z=\prod_{q=1}^{n} z_{q}$, we obtain from Theorem 1 of [5] the existence of a linear mapping $\gamma=\gamma_{\mathbf{z}}: \mathbb{R}^{n} \rightarrow \mathbb{R}^{n}$ satisfying

$$
\gamma\left(\mathbb{Z}^{n}\right)=\mathfrak{c} \quad \text { and } \quad \sup _{|\mathbf{t}|_{\infty} \leq 1}\left|\gamma^{(q)}(\mathbf{t})\right| \leq c_{10} d^{1 / 2} N \mathfrak{c}^{1 / n} z_{q} Z^{-1 / n} .
$$

This implies

$$
\begin{aligned}
& \sum_{\substack{\gamma \in \mathfrak{c} \\
z_{q}<\left|\gamma^{(q)}\right| \leq 2 z_{q}}}=\sum_{\substack{\mathbf{m} \in \mathbb{Z}^{n} \\
z_{q}<\left|\gamma^{(q)}(\mathbf{m})\right| \leq 2 z_{q}}} \int_{\mathbf{m}+[0,1]^{n}} d \mathbf{t} \\
& \leq \operatorname{Vol}\left(\mathbf{t} \in \mathbb{R}^{n}|| \gamma^{(q)}(\mathbf{t}) \mid \leq 2 z_{q}+O\left(d^{1 / 2} N \mathfrak{c}^{1 / n} z_{q} Z^{-1 / n}\right)\right) \\
& \ll \frac{1}{d^{1 / 2} N \mathfrak{c}} \prod_{q=1}^{n}\left(2 z_{q}+O\left(d^{1 / 2} N \mathfrak{c}^{1 / n} z_{q} Z^{-1 / n}\right)\right) \\
& \ll \frac{Z}{d^{1 / 2} N \mathfrak{c}}+d^{(n-1) / 2} .
\end{aligned}
$$

Since $\gamma \in \mathfrak{c}$ and $0<|\gamma|_{\infty} \leq M$ imply

$$
|N \gamma| \geq N \mathfrak{c} \quad \text { and } \quad\left|\gamma^{(q)}\right|=|N \gamma| \prod_{p \neq q}\left|\gamma^{(p)}\right|^{-1} \geq N \mathfrak{c} M^{1-n}
$$

we conclude that

$$
\begin{aligned}
& \sum_{\substack{\gamma \in \mathfrak{c} \\
0<|\gamma|_{\infty} \leq M}} \frac{1}{|N \gamma|} \leq \sum_{\substack{0 \leq k_{1}, \ldots, k_{n} \leq \frac{\log \left(M^{n-1 / N \mathfrak{c})}\right.}{\log 2} \\
\leq}} \sum_{\substack{\gamma \in \mathfrak{c} \\
M 2^{-k_{q}-1}<\left|\gamma^{(q)}\right| \leq M 2^{-k_{q}}}} \frac{1}{|N \gamma|} \sum_{\substack{\gamma \in \mathfrak{c} \\
0 \leq k_{1}, \ldots, k_{n} \leq \frac{\log \left(M^{n-1 / N \mathfrak{c})}\right.}{\log 2}}} \min \left(\frac{1}{N \mathfrak{c}}, M^{-n} 2^{\Sigma k_{q}+n}\right) \sum_{\substack{2^{-k_{q}-1}<\left|\gamma^{(q)}\right| \leq M 2^{-k_{q}}\\
}} \sum_{0 \leq k_{1}, \ldots, k_{n} \leq \frac{\log \left(M^{n-1 / N \mathfrak{c})}\right.}{\log 2}} \min \left(\frac{1}{N \mathfrak{c}}, M^{-n} 2^{\Sigma k_{q}}\right)\left(\frac{M^{n} 2^{-\Sigma k_{q}}}{d^{1 / 2} N \mathfrak{c}}+d^{(n-1) / 2}\right) \\
& \ll \log ^{n}\left(M^{n} / N \mathfrak{c}\right)\left(d^{-1 / 2} N \mathfrak{c}^{-1}+d^{(n-1) / 2} N \mathfrak{c}^{-1}\right) .
\end{aligned}
$$

Let $G(\gamma)$ denote the Gaussian sum $\sum_{\varrho \bmod \mathfrak{f}} \chi(\varrho) e(S(\gamma \varrho)), \gamma \in 1 /(\mathfrak{d} \mathfrak{f})$. Since $\chi$ is primitive one has the well-known 
LEMMA 6.

$$
|G(\gamma)|= \begin{cases}0, & (\gamma \mathfrak{d} \mathfrak{f}, \mathfrak{f}) \neq 1 \\ N \mathfrak{f}^{1 / 2}, & (\gamma \mathfrak{d} \mathfrak{f}, \mathfrak{f})=1\end{cases}
$$

Proof of Theorem 1. One has

$$
\begin{aligned}
& \sum_{y_{q}<\nu(q) \leq y_{q}+x_{q}} \chi(\nu) \\
& =\sum_{\nu_{0} \bmod \mathfrak{f}} \chi\left(\nu_{0}\right)\left|\left\{\nu \in \mathbb{Z}_{K} \mid \nu \equiv \nu_{0} \bmod \mathfrak{f}, y_{q}<\nu^{(q)} \leq y_{q}+x_{q}\right\}\right| \\
& =\left(\sum_{\nu_{0} \bmod \mathfrak{f}} \chi\left(\nu_{0}\right)\right) \frac{X}{d^{1 / 2} N \mathfrak{f}} \\
& \quad+\sum_{\substack{\mathbf{n} \in \mathbb{Z}^{n} \\
0<|\mathbf{n}|_{\infty} \leq N}} b_{\mathbf{n}} \sum_{\nu_{0} \bmod \mathfrak{f}} \chi\left(\nu_{0}\right) e\left(S\left(\eta(\mathbf{n}) \beta \nu_{0}\right)\right)+O(1)
\end{aligned}
$$

by Theorem 3, with $N:=c_{8}(d N \mathfrak{f} X)^{c_{9}} \geq N \mathfrak{f}^{3}$. Analogously to (11),

$$
\max _{|\mathbf{t}|_{\infty} \leq 1}|\eta(\mathbf{t})| \leq c_{11} d^{n-1} N \mathfrak{f}^{-1 / n}
$$

follows. This yields

(13) $\quad\left\{\left.\eta(\mathbf{n})\left|\mathbf{n} \in \mathbb{Z}^{n}, 0<\right| \mathbf{n}\right|_{\infty} \leq N\right\} \subset\left\{\eta \in 1 /\left.(\mathfrak{d} \beta \mathfrak{f})|0<| \eta\right|_{\infty} \leq N^{2}\right\}$

since $N \geq c_{12} d^{n-1}$.

From Lemma 6 one infers

$$
\begin{aligned}
\sum_{\substack{\mathbf{n} \in \mathbb{Z}^{n} \\
0<|\mathbf{n}|_{\infty} \leq N}}\left|b_{\mathbf{n}}\right|\left|\sum_{\nu_{0} \bmod \mathfrak{f}} \chi\left(\nu_{0}\right) e\left(S\left(\eta(\mathbf{n}) \beta \nu_{0}\right)\right)\right| \\
\ll \frac{N \mathfrak{f}^{1 / 2}}{d^{1 / 2} N \beta \mathfrak{f}} \sum_{\substack{\eta \in 1 /(\mathfrak{d} \beta \mathfrak{f}) \\
0<|\eta|_{\infty} \leq N^{2} \\
(\eta \mathfrak{d} \beta \mathfrak{f}, \mathfrak{f})=1}} \prod_{q=1}^{n} \min \left(\frac{1}{\left|\eta^{(q)}\right|}, X^{1 / n}\right) \\
\ll \frac{1}{d^{1 / 2} N \beta N \mathfrak{f}^{1 / 2}} \sum_{\substack{\eta \in 1 /(\mathfrak{o} \beta \mathfrak{f}) \\
0<|\eta|_{\infty} \leq N^{2}}} \frac{1}{|N \eta|} \ll d^{n / 2} N \mathfrak{f}^{1 / 2}(\log N)^{n}
\end{aligned}
$$

by Lemma 5 .

So Theorem 1 follows directly for $N \mathfrak{f}^{1 / 2} \leq X$ (implying $\log (d N \mathfrak{f} X) \ll$ $\log (d X)$ ); otherwise it is trivial (use Theorem 1 with $\mathfrak{f}=\mathbb{Z}_{K}$ ).

The proof of Proposition 1 follows in the same way.

4. Lower bounds. To derive lower bounds we fix $\nu_{0} \in \mathbb{Z}_{K}$, replace $\beta$ by 1 and work with the Fourier series of $F\left(\alpha^{-1}\left(\nu_{0}\right), \mathbf{z} ; \mathbf{v}, \alpha\right)$ with respect to $\mathbf{z}$. 
From (6) follows the existence of $\mathbf{w} \in \mathbb{R}^{n},|\mathbf{w}|_{\infty} \ll d^{1 / 2} N \mathfrak{f}^{1 / n}$, satisfying

$$
\Delta:=\mathbf{w}+\alpha\left([0,1]^{n}\right) \subset \mathbb{R}_{+}^{n} .
$$

In $L^{2}(\Delta)$,

$$
F\left(\alpha^{-1}\left(\nu_{0}\right), \cdot ; \mathbf{v}, \alpha\right)=\sum_{\gamma \in 1 /(\mathfrak{d} \mathfrak{f})} c_{\gamma} e(-\langle\gamma, \cdot\rangle)
$$

holds where the coefficients are given by

$$
\begin{aligned}
c_{\gamma} & =c_{\gamma}\left(\nu_{0}, \mathbf{v}, \alpha\right)=\frac{1}{\operatorname{Vol} \Delta} \int_{\Delta} F\left(\alpha^{-1}\left(\nu_{0}\right), \mathbf{z}\right) e(-\langle\gamma, \mathbf{z}\rangle) d \mathbf{z} \\
& =\frac{e\left(-S\left(\gamma \nu_{0}\right)\right)}{d^{1 / 2} N \mathfrak{f}} \int_{\Delta} \sum_{\substack{\nu \in \mathfrak{f} \\
z_{p}<\nu^{(p)}+\nu_{0}^{(p)} \leq z_{p}+v_{p}}} e\left(\left\langle\gamma, \nu+\nu_{0}-\mathbf{z}\right\rangle\right) d \mathbf{z} \\
& =\frac{e\left(-S\left(\gamma \nu_{0}\right)\right)}{d^{1 / 2} N \mathfrak{f}} \sum_{\substack{\nu \in \mathbb{Z}_{K} \\
\nu \equiv \nu_{0} \bmod \mathfrak{f}}} \Delta \cap\left\{\mathbf{z} \mid 0<\nu^{(p)}-z_{p} \leq v_{p}\right\} \\
& \left.\left.=\frac{e\left(-S\left(\gamma \nu_{0}\right)\right)}{d^{1 / 2} N \mathfrak{f}} \int_{\left\{\mathbf{z} \mid 0<z_{p} \leq v_{p}\right\}} e(\langle\gamma, \mathbf{z}\rangle) d \mathbf{z}-\mathbf{z}\right\rangle\right) d \mathbf{z} \\
& =\frac{e\left(-S\left(\gamma \nu_{0}\right)\right)}{d^{1 / 2} N \mathfrak{f}} \prod_{p=1}^{n} \int_{0}^{v_{p}} e\left(\gamma^{(p)} t_{p}\right) d t_{p} \\
& = \begin{cases}\frac{1}{(2 \pi i)^{n}} \frac{e\left(-S\left(\gamma \nu_{0}\right)\right)}{d^{1 / 2} N \mathfrak{f}} \frac{1}{N \gamma} \prod_{p=1}^{n}\left(e\left(\gamma^{(p)} v_{p}\right)-1\right), \quad \gamma \neq 0, \\
\frac{X}{d^{1 / 2} N \mathfrak{f}}, & \gamma=0 .\end{cases}
\end{aligned}
$$

Remark. $c_{\gamma}\left(\nu_{0}, \mathbf{v}, \alpha\right) e\left(S\left(\eta \nu_{0}\right)\right)=\overline{a_{\eta^{-1}(\gamma)}(\mathbf{z}, \mathbf{v}, \alpha)} e(-\langle\gamma, \mathbf{z}\rangle)$.

Proposition 4. Let $\gamma_{0} \in 1 /(\mathfrak{d} \mathfrak{f})-\{0\}$ satisfy $\left(\gamma_{0} \mathfrak{d} \mathfrak{f}, \mathfrak{f}\right)=1$. For any $\mathbf{y} \in \mathbb{R}^{n}$ there is an $\mathbf{x} \in \mathbb{R}_{+}^{n},|\mathbf{x}|_{\infty} \ll\left|1 / \gamma_{0}\right|_{\infty}+d^{1 / 2} N \mathfrak{f}^{1 / n}$, satisfying

$$
\left|\sum_{y_{q}<\nu^{(q)} \leq y_{q}+x_{q}} \chi(\nu)-E(\chi) X\right| \geq \frac{1}{(2 \pi)^{n} d^{1 / 2}} \frac{1}{N \mathfrak{f}^{1 / 2}\left|N \gamma_{0}\right|} .
$$

Proof. One has

$$
\begin{aligned}
h(\mathbf{z}): & =\sum_{z_{q}+y_{q}<\nu^{(q)} \leq y_{q}+z_{q}+v_{q}} \chi(\nu)-E(\chi) \prod_{q=1}^{n} v_{q} \\
& =\sum_{\nu \bmod \mathfrak{f}} \chi(\nu) \sum_{\gamma \in 1 /(\mathfrak{d} \mathfrak{f})-\{0\}} c_{\gamma}(\nu, \mathbf{v}, \alpha) e(-\langle\gamma, \mathbf{z}+\mathbf{v}\rangle)
\end{aligned}
$$

in $L^{2}(\Delta)$ by $(14)$. 
Parseval's equation and (15) lead to

$$
\begin{aligned}
\max _{\mathbf{z} \in \Delta}|h(\mathbf{z})|^{2} & \geq \frac{1}{\operatorname{Vol} \Delta} \int_{\Delta}|h(\mathbf{z})|^{2} d \mathbf{z} \\
& =\sum_{\gamma \in 1 /(\mathfrak{d f})-\{0\}}\left|\sum_{\nu \bmod \mathfrak{f}} c_{\gamma}(\nu, \mathbf{v}, \alpha) \chi(\nu)\right|^{2} \\
& \geq \frac{1}{\left(4 \pi^{2}\right)^{n}} \frac{1}{d N \mathfrak{f}^{2}} \frac{1}{\left|N \gamma_{0}\right|^{2}}\left|G\left(-\gamma_{0}\right)\right|^{2} \prod_{p=1}^{n}\left|e\left(\gamma_{0}^{(p)} v_{p}\right)-1\right|^{2}
\end{aligned}
$$

The product is $4^{n}$ if we choose $v_{p}$ to be $\left(2\left|\gamma_{0}^{(p)}\right|\right)^{-1}$.

By use of Lemma 6 we obtain the existence of $\mathbf{z} \in \Delta$ (thus $0<z_{q} \ll$ $\left.d^{1 / 2} N \mathfrak{f}^{1 / n}\right)$ satisfying

$$
\begin{aligned}
& \frac{1}{\pi^{n} d^{1 / 2} N \mathfrak{f}} \frac{1}{\left|N \gamma_{0}\right|} N \mathfrak{f}^{1 / 2} \leq|h(\mathbf{z})| \\
& =\mid \sum_{z_{q}+y_{q}<\nu^{(q)} \leq y_{q}+z_{q}+v_{q}} \chi(\nu) \\
& \quad-E(\chi) \operatorname{Vol}\left(\mathbf{v} \in \mathbb{R}^{n} \mid y_{q}+z_{q}<v_{q} \leq y_{q}+z_{q}+v_{q}\right) \mid \\
& =\mid \sum_{c_{1}, \ldots, c_{n}=0}^{1}(-1)^{n-\Sigma c_{q}}\left(\sum_{y_{q}<\nu^{(q)} \leq y_{q}+z_{q} c_{q}+v_{q}} \chi(\nu)\right. \\
& \left.-E(\chi) \operatorname{Vol}\left(\mathbf{v} \in \mathbb{R}^{n} \mid y_{q}<v_{q} \leq y_{q}+z_{q} c_{q}+v_{q}\right)\right) \mid .
\end{aligned}
$$

So at least one of the $2^{n}$ values of $\left(z_{q} c_{q}+v_{q}\right)_{q=1}^{n}$ can be chosen to be $\mathbf{x}$.

Proof of Theorem 2. The ideal class generated by of contains at least $2 \omega(\mathfrak{f})$ prime ideals of norm less than $c_{13}(K) \omega(2 \mathfrak{f}) \log (\omega(6 \mathfrak{f}))$. Thus one of these ideals, say $\mathfrak{p}$, does not divide $\mathfrak{f}$. Any generator $\gamma_{0}$ of the principal ideal $\mathfrak{p} /(\mathfrak{d} \mathfrak{f})$ satisfying

$$
\left|\gamma_{0}^{(q)}\right| \ll_{K}\left|N \gamma_{0}\right|^{1 / n} \ll_{K} \frac{\omega(2 \mathfrak{f}) \log (\omega(6 \mathfrak{f}))}{N \mathfrak{f}} \quad \text { (see e.g. (81) of [9]) }
$$

is admissible in Proposition 3 since

$$
\left(\gamma_{0} \mathfrak{d} \mathfrak{f}, \mathfrak{f}\right)=(\mathfrak{p}, \mathfrak{f})=1
$$

This proves Theorem 2. If $\mathfrak{d} \mathfrak{f}=(\varrho)$ is principal one applies Proposition 4 with $\gamma_{0}=1 / \varrho$.

Acknowledgements. The author would like to thank Acta Arithmetica's referee for fruitful hints giving Lemma 1 its final form. 


\section{References}

[1] K. M. Bartz, On a theorem of A. V. Sokolovskiu, Acta Arith. 34 (1978), 113-126.

[2] G. H. Hardy and J. E. Littlewood, Some problems of Diophantine approximation: The lattice-points of a right-handed triangle, Proc. London Math. Soc. (2) 20 (1921), $15-36$.

[3] J. G. Hinz, Character sums in algebraic number fields, J. Number Theory 17 (1983), $52-70$.

[4] K. C. Lee, On the average order of characters in totally real algebraic number fields, Chinese J. Math. 7 (1979), 77-90.

[5] K. Mahler, Inequalities for ideal bases in algebraic number fields, J. Austral. Math. Soc. 4 (1964), 425-448.

[6] H. L. Montgomery and R. C. Vaughan, Mean values of character sums, Canad. J. Math. 31 (1979), 476-487.

[7] G. Pólya, Über die Verteilung der quadratischen Reste und Nichtreste, Göttinger Nachr. (1918), 21-29.

[8] U. Rausch, Character sums in algebraic number fields, to appear.

[9] G. J. Rieger, Verallgemeinerung der Siebmethode von A. Selberg auf algebraische Zahlkörper. III, J. Reine Angew. Math. 208 (1961), 79-90.

[10] M. M. Skriganov, Lattices in algebraic number fields and uniform distribution mod 1, Leningrad Math. J. 1 (1990), 535-558.

[11] D. C. Spencer, The lattice points of tetrahedra, J. Math. Phys. 21 (1942), 189-197.

[12] T. Tatuzawa, Fourier analysis used in analytic number theory, Acta Arith. 28 (1975), 263-272.

ZUM DONNERBERG 14

D-W-3554 GLADENBACH, GERMANY

Received on 19.6.1992

and in revised form on 1.2.1993 